\title{
Editorial
}

\section{Magnetic and Transport Properties Based on Transition-Metal Compounds}

\author{
Ran Ang, ${ }^{1}$ Hechang Lei, ${ }^{2}$ Shoubao Zhang, ${ }^{3}$ and Xuan Luo ${ }^{4}$ \\ ${ }^{1}$ Key Laboratory of Materials Physics, Institute of Solid State Physics, Chinese Academy of Sciences, Hefei 230031, China \\ ${ }^{2}$ Frontier Research Center, Materials and Structures Laboratory, Tokyo Institute of Technology, 4259 Nagatsuta, Midori, \\ Yokohama 226-8503, Japan \\ ${ }^{3}$ Advanced Solid State Chemistry Laboratory, Institute for Chemical Research, Kyoto University, Gokasho, Uji, Kyoto 611-0011, Japan \\ ${ }^{4}$ Laboratory for Pohang Emergent Materials, Department of Physics, Pohang University of Science and Technology, \\ Pohang 790-784, Republic of Korea
}

Correspondence should be addressed to Ran Ang; rang@issp.ac.cn

Received 20 February 2014; Accepted 20 February 2014; Published 7 May 2014

Copyright (C) 2014 Ran Ang et al. This is an open access article distributed under the Creative Commons Attribution License, which permits unrestricted use, distribution, and reproduction in any medium, provided the original work is properly cited.

So far, transition-metal compounds have triggered a tremendous wave of excitement in the scientific community due to their rich physical properties. It is extremely important to understand and realize the $d$-orbital state to tune the fundamental physical properties (i.e., magnetic properties and electrical and thermal transport) of transition-metal compounds as well as their applications. The selected topics and papers include the typical magnetism and transport properties; thus it is worth sharing with many readers. This special issue contains seven papers. The detailed information is as follows.

In the paper "Tuning of transport and magnetic properties in epitaxial $\mathrm{LaMnO}_{3+\delta}$ thin films," J. Chen et al. present the effect of compressive strain induced by substrate on the transport and magnetic properties of $\mathrm{LaMnO}_{3+\delta}$ thin films. It is found that the insulator-metal transition, charge/orbital ordering transition, and paramagnetic-ferromagnetic transition are suppressed by the compressive strain. In particular, the electronic and magnetic transition temperatures decrease with increasing the compressive strain. The present results reveal that the lattice degree of freedom plays a key role to control the transport and magnetic properties of the strongly correlated $\mathrm{LaMnO}_{3+\delta}$ thin films.

In the paper entitled "Aging effect on electrical conductivity of pure and $\mathrm{Al}$-doped $\mathrm{YBa}_{2} \mathrm{Cu}_{3} \mathrm{O}_{7-\delta}$ single crystals with a given topology of planar defects," R. V. Vovk et al. present the study of the conducting properties in the basal ab plane of pure and $\mathrm{Al}$-doped $\mathrm{YBa}_{2} \mathrm{Cu}_{3} \mathrm{O}_{7-\delta}$ single crystals before and after long-time exposure in air atmosphere. It is shown that prolonged aging leads to an increase of the density of effective scattering centers for the normal carriers. The aluminum doping has been revealed to partially slow down the degradation of the conducting properties in process of aging. The excess conductivity, $\Delta \sigma(T)$, has been found to obey exponential dependence in the broad temperature range $T_{c}<T<T^{*}$. In the pseudogap regime, the mean-field transition temperature $T^{*}$ and the $3 \mathrm{D}-2 \mathrm{D}$ crossover point in the excess conductivity have been quantified. Near the critical temperature, $\Delta \sigma(T)$ is described well within the AslamazovLarkin theoretical model. Herewith, both aluminum doping and prolonged aging have been found to essentially expand the temperature interval of implementation of the pseudogap state, thus narrowing the linear section in the dependence $\rho_{a b}(T)$.

In the paper "Magnetism and microstructure characterization of phase transitions in a steel," M. Güler presents the study of phase transitions in a low carbon steel according to the existing phases and their magnetism. Scanning electron microscope examinations showed that pure state of the steel was fully in the ferrite phase with equiaxed grains. Moreover, subsequent heat treatments on the studied steel also ensured the formation of austenite and followed pearlite 
phases. Mössbauer spectroscopy of these phases appeared as a paramagnetic single-line absorption peak for the austenite phase and ferromagnetic six-line spectra for both ferrite and pearlite phases. From Mössbauer data the determined the internal magnetic fields of ferrite and pearlite phases were as 32.2 Tesla and 31.3 Tesla, respectively.

In the paper "Defects induced room temperature ferromagnetism in $\mathrm{ZnO}$ thin films," X. Zhang et al. prepared polycrystalline $\mathrm{ZnO}$ thin films by the cosputtering method under different oxygen partial pressures. They found that the films deposited in pure argon gas exhibited ferromagnetism, whereas other films deposited under different oxygen partial pressures were diamagnetism. The study indicated that $\mathrm{Zn}$ interstitial may play an important role in triggering magnetic order in the $\mathrm{ZnO}$ thin films by inducing an alteration of electronic configuration.

In the paper "Transport, magnetic, and thermal properties of $\mathrm{La}_{0.7} \mathrm{Ca}_{0.24} \mathrm{Sr}_{0.06} \mathrm{MnO}_{3}$ single crystal," T. M. Tank et al. present the transport, magnetic, and thermal properties of $\mathrm{La}_{0.7} \mathrm{Ca}_{0.24} \mathrm{Sr}_{0.06} \mathrm{MnO}_{3}$ single crystal. They found that the floating zone (FZ) method was most suitable for the growth of CMR manganite. This study reveals that the magnetic transition temperature increased substantially to $277 \mathrm{~K}$ which indicates the onset of long range ordering and large TCR values are a highly desirable goal in the context of the development of highly responsive bolometer device.

In the paper entitled "Oxygen defects mediated magnetism of $\mathrm{Ni}$ doped $\mathrm{ZnO}$," W. J. Liu et al. synthesize $\mathrm{Ni}$ doped $\mathrm{ZnO}$ nanoparticles by a solution route and they are annealed in $\mathrm{O}_{2}$, air, and Ar gas flow. Annealed $\mathrm{ZnO}$ samples show ferromagnetism. They discussed the origin of ferromagnetism based on magnetization and photoluminescence spectroscopy.

In another paper, J. Yun et al. present "Magnetic properties of well-aligned $\mathrm{ZnO}$ nanorod arrays grown by a simple hydrothermal reaction." Well-aligned $\mathrm{ZnO}$ nanorod arrays with room temperature ferromagnetism were prepared by hydrothermal method. The $\mathrm{ZnO}$ nanorods show (002) direction with $c$-axis perpendicular to the substrate surface. They discussed the nature of the ferromagnetism on the term of photoluminescence spectrum and the first-principles calculations.

\section{Acknowledgments}

We would like to thank the authors for their significant contributions and patient assistance. We also warmly thank all reviewers on these papers.

Ran Ang

Hechang Lei

Shoubao Zhang

Xuan Luo 

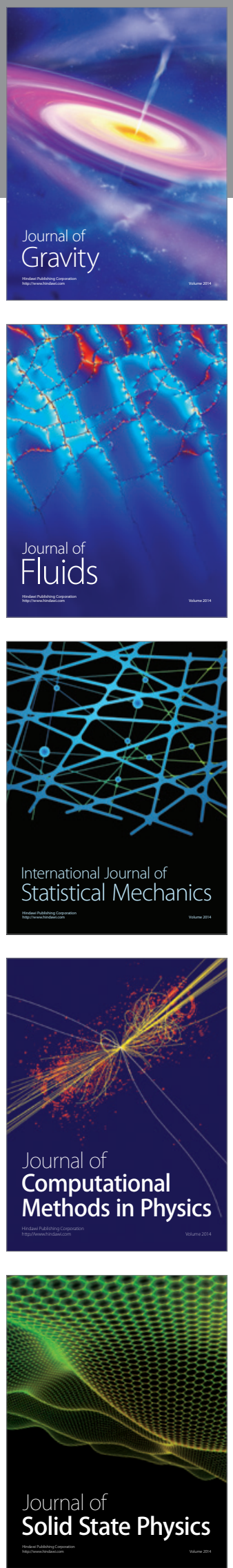

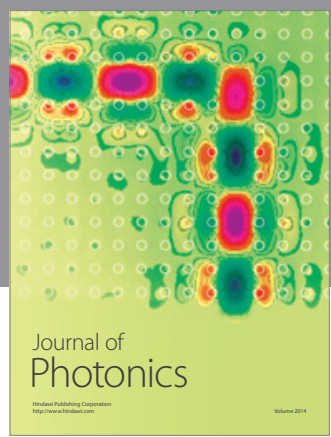

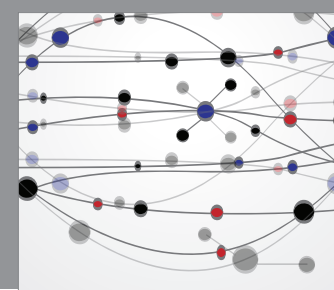

The Scientific World Journal

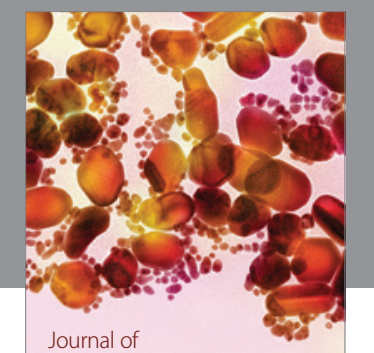

Soft Matter
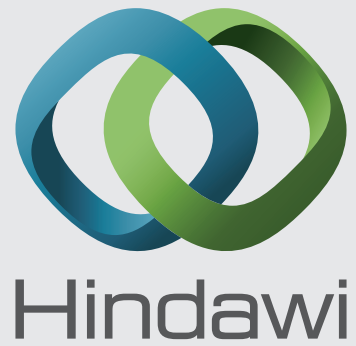

Submit your manuscripts at

http://www.hindawi.com
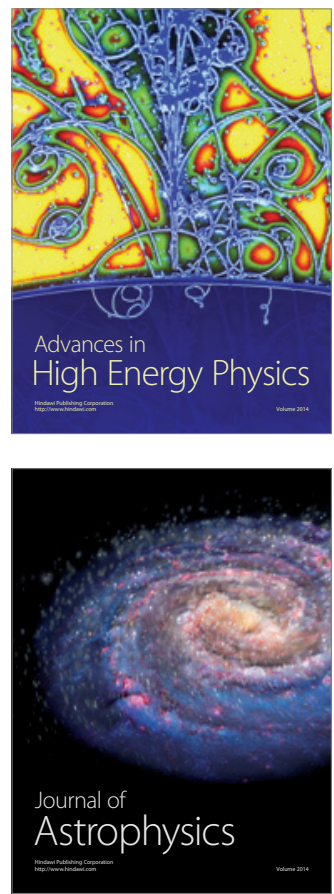
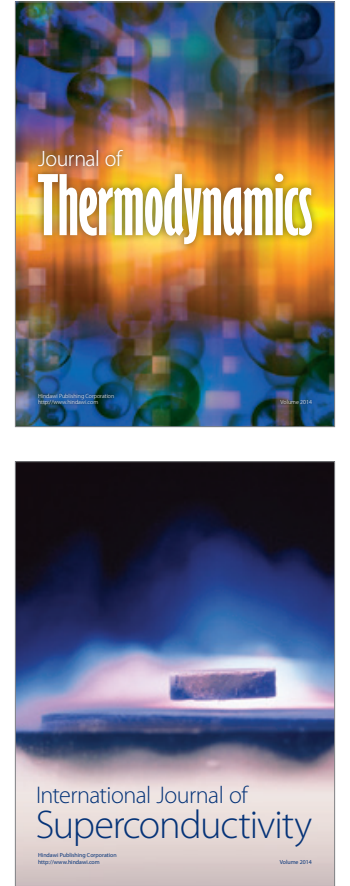
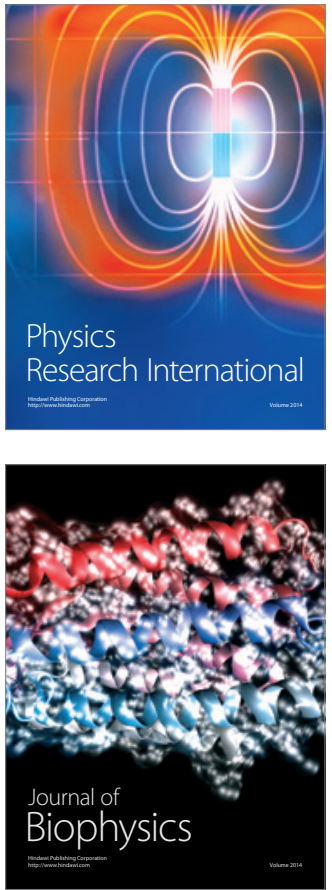
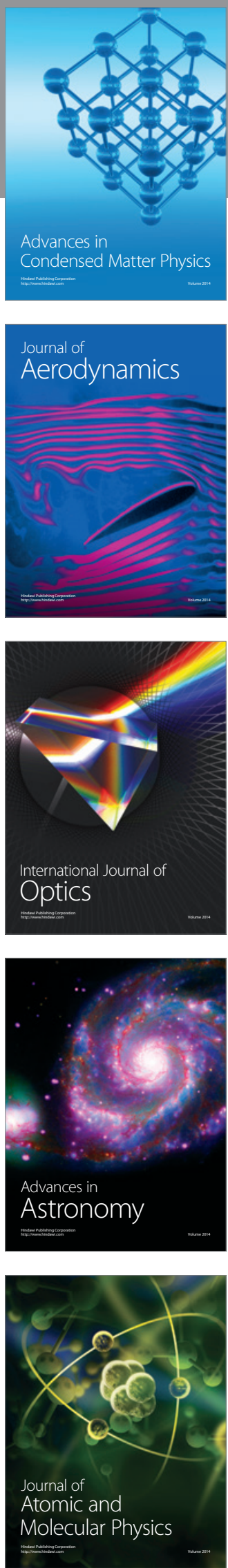